\title{
The impact of alcohol use in Schizophrenia
}

L. Linhares¹, M.J. Gonçalves¹, C. Sereijo¹, S. Pombo², F. Ismail11.

${ }^{1}$ Centro Hospitalar Universitário Lisboa Norte, Department of Psychiatry, Lisbon, Portugal.

2Faculty of Medicina - University of Lisbon, Clínica Universitária de Psiquiatria, Lisbon, Portugal.

$19^{\text {TH }}$ WPA WORLD CONGRESS OF PSYCHIATRY UISBON, 21-24 AUGUST, 2019

Comorbidity in psychiatry is common and may require a different approach beyond the simple sum of each disorder treatment. Regarding

Substance Use Disorder, alcohol is widely distributed by several psychiatric conditions. During the last two decades, numerous studies have reported an increase in alcohol consumption among schizophrenic patients. Comorbidity ranges from 35 to $80 \%$.

\section{Methods:}

Non-systematic review of the literature using PubMed database. Research of articles with the keywords: "schizophrenia", "alcohol", "comorbidity", published in last ten years and selected by its scientific relevance.

\section{PublMed}

\section{Results:}

\section{Impact}

- Unemployment

- Exacerbation of psychiatric symptoms

- Greater use of emergency services

- Increased number of psychiatric hospitalization

- Earlier rehospitalizations compared with ones with schizophrenia with no alcohol consumption

- Higher levels of cognitive impairment compared to ones without schizophrenia

- Higher levels of suicide attempts

- Structural brain changes not attributable to schizophrenia per se

- Higher numbers of suicide attempts
- Male patients with schizophrenia with higher levels of comorbid alcohol disorder

- High rates of alcoholism heritage (higher than in general population)

- Neuroticism features during childhood related in favor of less comorbility in adulhood in schizophrenic patients

\section{What patients say?}

Used as a way of socialization

Used as self-medication for depression, anxiety and psychotic symptoms

\section{Is this population vulnerable?}

Some studies suggest that impulsivity and the seeking for sensations present among patients with schizophrenia may increase the risk for alcohol use and dependence. On the other hand, this group of patients presents an increased stimulatory response to alcohol, leading to an increased risk of dependence. Also, alcohol appears to cause structural brain changes that are not attributable to schizophrenia per se. Patients with schizophrenia are a population at risk for alcohol consumption.

\section{Conclusion:}

- The treatment of both disorders is crucial to prevent this comorbidity consequences'.

- Combined treatment with targeting both alcohol consumption and psychiatric disorder gives better results than addressing each disorder separately.

- Although the impact of alcohol on this population is well known, there are still few studies evaluating the various psychological interventions that could improve the treatment and prognosis of this comorbidity.

\section{References:}

Thornton LK, Baker AL. The importance of investigating alcohol use among people with schizophrenia. Acta Psychiatry Scand. 2013 Jul;128(1):96. Bennett, ME et all, Treatment of substance use disorders in schizophrenia, Am J Drug Alchohol Abuse 2017 Jul;43(4):377-390

Bennett, ME et all, Treatment of substance use disorders in schizophrenia, Am J Drug Alchohol Abuse 2017 Jul;43(4):377-390
Morgan VA, Waterreus A, Jablensky A et al., People living with psychotic illness in 2010: the second Australian national survey of psychosis. Aust N Z J Psychiatry 2012;46:735-752

Ziedonis D, Nickou C. Substance abuse in patients with schizophrenia. In: Hwang MY, Bermanzohn PC, eds. Schizo- phrenia and comorbid conditions: Diagnosis and treatment. Washington: American Psychiatric Press. 2001: 187-222.

Baker A, Hiles S, Thornton L, Hides L, Lubman DI. A systematic review of psychological interventions for exces- sive alcohol consumption among people with psychotic disorders. Acta Psychiatr Scand 2012;126:243-255.

Lin C-H, Huang C-J, Huang Y-H, Chen C-C. Time to rehos- pitalization of schizophrenia patients with alcohol use disorders. Acta Psychiatr Scand 2013;128:94-95 